\title{
Prevención de la isoinmunización materna al RhD, con $\gamma$-globulina anti-D
}

\author{
Héctor Alfredo Baptista-González, M.C., ${ }^{(1)}$ Fany Rosenfeld-Mann, Q .C.B., ${ }^{(1)}$ \\ Teresita Leiss-Márquez, M.C.(2)
}

\section{Baptista-González HA, Rosenfeld-Mann F, Leiss-Márquez T. \\ Prevención de la isoinmunización materna al RhD, con g-globulina anti-D. Salud Publica Mex 2001;43:52-58.}

El texto completo en inglés de este artículo está disponible en: http://www.insp.mx/salud/index.html

\section{Resumen}

Objetivo. Presentar la experiencia institucional en la prevención de la isoinmunización al RhD, mediante el empleo de $150 \mu \mathrm{g}$ de $\gamma$-globulina anti-D en las mujeres Rh negativo. Material y métodos. Se registraron los antecedentes inmunohematológicos de los casos consecutivos de todas las mujeres Rh negativo que acudieron, para su atención médica, al Instituto $N$ acional de Perinatología entre 1982 y 1995. A las mujeres con riesgo de isoinmunización se les aplicó $150 \mu \mathrm{g}$ de $\gamma$-globulina anti-D, con fines preventivos. Resultados. En el periodo de estudio ingresaron 4857 mujeres Rh negativo (4.85\% del total de mujeres), de las cuales $629(13.0 \%)$, presentaron isoinmunización al RhD. De estas últimas, 542 (86.2\%) ya se encontraron isoinmunizadas desde antes de su ingreso al Instituto. En 22 casos (3.5\%), la isoinmunización ocurrió a pesar de que recibieron la dosis adecuada de $\gamma$-globulina anti D. De las 2605 pacientes (53.6\%) sometidas a prevención, a 2039 se les aplicó una sola dosis, y a 475, hasta dos dosis. En 22 casos se documentó la falla de la prevención; sin embargo, en cuatro de ellos, se registraron embarazos múltiples, y los restantes 18 presentaron patología obstétrica asociada. Conclusiones. Mediante este programa de prevención, consistente en administrar $150 \mu \mathrm{g}$ de $\gamma$-globulina anti-D por dosis, es posible reducir la iso inmunización a menos de un caso por cada 1000 mujeres. Los fracasos en la prevención de la isoinmunización se asociaron a condiciones obstétricas agregadas y al incumplimiento de las guías o lineamientos del programa. El texto completo en inglés de este artículo está disponible en: http://www.insp.mx/salud/index.html

Palabras clave: isoinmunización $\mathrm{Rh} /$ prevención \& control; México

\author{
Baptista-González HA, Rosenfeld-Mann F, \\ Leiss-Márquez T. \\ Prevention of maternal RhD isoimmunization \\ with anti-D gamma globulin. \\ Salud Publica Mex 2001;43:52-58. \\ The English version of this paper \\ is available at: http://www.insp.mx/salud/index.html
}

\begin{abstract}
A bstract
Objective. To report our experience in preventing $\mathrm{RhD}$ maternal isoimmunization by using anti-D gamma globulin among Rh-negative women. Material and methods. Between 1982 and 1995, immunologic and hematologic data were collected from all Rh-negative women seen at Mexico's $\mathrm{N}$ ational Perinatology Institute. Women at risk of $\mathrm{Rh}$ iso immunization were given a prophylactic dose of $150 \mu \mathrm{g}$ of anti-D gamma globulin. Results. A total of $4857 \mathrm{Rh}$ negative women were seen during the study period $(4.85 \%$ of the total population of women seen at the Institute), $629(13.0 \%)$ of whom developed RhD isoimmunization; $542(86.2 \%)$ of these women were already iso immunized when first seen at our Institute. Twenty-two women (3.5\%) developed isoimmunization even after receiving a proper dose of anti-D gamma globulin. Prophylaxis was given to 2605 women $(53.6 \%) ; 2039$ received a single dose, and 475 two doses. Prophylaxis failed in 22 cases; four were women with multiple pregnancy and 18 developed obstetric pathologic conditions. Conclusions. The use of anti-D gamma globulin resulted in a reduction of maternal $\mathrm{Rh}$ iso immunization to less than one case per 1000 women. Failures to prevent iso immunization were associated to additional obstetric conditions and to lack of adherence to prevention guidelines. The English version of this paper is available at: http://www.insp.mx/salud/index.html
\end{abstract}

Key words: Rh isoimmunization/prevention \& control; Mexico

(1) Coordinación de Hematología Perinatal, Instituto N acional de Perinatología (IN Per), México.

(2) Departamento de Medicina Fetal, IN Per, México.

Fecha de recibido: 23 de mayo de 2000 - Fecha de aprobado: 7 de noviembre de 2000

Solicitud de sobretiros: Dr. Héctor A. Baptista González. Hematología Perinatal, Subdirección de Investigación Clínica, Instituto N acional de Perinatología. Primer piso de la Torre de Investigación. Montes U rales 800, Lomas Virreyes, Delegación Miguel Hidalgo, 11000 México, D.F. Correo electrónico: baptista@ infosel.net.mx 
T a incidencia de la isoinmunización materna al $\mathrm{Rh}$, depende de la frecuencia génica del $\mathrm{Rh}$ en la población de estudio. Para México se estima que aproximadamente $1 \%$ de la población indígena y cerca de $3 \%$ de la población mestiza es $\mathrm{RhD}$ negativo. ${ }^{1}$ Existen amplias variaciones locales derivadas de la mezcla génica, ya que el fenotipo Rh negativo no es de origen amerindio. ${ }^{2}$

El riesgo para la salud perinatal de las mujeres Rh negativo, ocurre cuando están embarazadas y su feto es Rh positivo. Así, si no se toman las medidas de prevención correspondientes, entre 1 y $2 \%$ de estas mujeres resultarán isoinmunizadas en la etapa prenatal; aproximadamente $5-15 \%$ presentarán esta alteración, al momento del nacimiento de su bebé, y de 3 a $6 \%$ lo harán después de un aborto. ${ }^{3}$ No obstante, la amenaza de aborto rara vez se asocia a la isoinmunización, pues en menos de $10 \%$ de los casos se documenta hemorragia fetomaterna significativa. ${ }^{4} \mathrm{Sin}$ embargo, entre 2 y $5 \%$ de las pacientes se isoinmunizarán después de una amniocentesis o una aspiración de vellosidades coriónicas. ${ }^{5}$ Con la cordocentesis no se tiene establecida con precisión la magnitud del riesgo de sensibilización. ${ }^{6}$ Finalmente, el impacto de la isoinmunización se refleja en estimaciones de que aproximadamente $25 \%$ de los hijos de las mujeres afectadas fallecen en la etapa perinatal. ${ }^{7,8}$

La información sobre la enfermedad hemolítica por anti-D es escasa en México; ${ }^{9}$ aunque se sabe que la isoinmunización apenas representa $0.33 \%$ de las tasas de mortalidad perinatal, ${ }^{10}$ se desconoce su prevalencia. Sin embargo, se tienen informaciones de que en población caucásica la probabilidad de isoinmunización materna con fetos $\mathrm{Rh}$ incompatibles ocurre entre 9 y $22 \%$ de las mujeres en riesgo. $3,11,12$ A pesar de los serios problemas de subregistro que existe en los informes hospitalarios, ${ }^{13}$ se sabe que la prevalencia de isoinmunización al $\mathrm{RhD}$ ha declinado significativamente, a partir de la introducción de nuevas modalidades de tratamiento en la década de los setenta. Así, entre 1970 y 1979, en mujeres sajonas, la tasa cruda de isoinmunización disminuyó de 9.1-10.3 a 1.4 casos por 1000 nacimientos. ${ }^{14-17}$

La acción preventiva más importante para evitar la sensibilización materna, consiste en: a) detectar la incompatibilidad sanguínea y a la vez identificar el grupo sanguíneo $\mathrm{ABO}$ y el $\mathrm{RhD}$; b) identificar la existencia de la isoinmunización mediante la detección de anticuerpos antieritrocitarios irregulares en el suero materno, con la prueba de la antiglobulina indirecta -Coombs indirecto- ${ }^{18,19}$ y c) aplicar la $\gamma$-globulina anti-D junto con la disminución de la tasa de fertilidad. $18,20-22$
A pesar de los beneficios comprobados de la $\gamma$-globulina anti-D, en nuestro país persiste la isoinmunización de mujeres $\mathrm{Rh}$ negativo por razones socioeconómicas (por el alto costo del producto y la baja disponibilidad en el mercado), médicas (criterio e indicaciones para su empleo) y biológicas (respuesta inmunológica individual, momento de la sensibilización, etc.). ${ }^{23}$ Un factor que puede disminuir la persistencia de la isoinmunización materna, es la adecuada organización de los servicios de atención médica, pues la experiencia internacional demuestra las ventajas que se derivan de agrupar y organizar los recursos humanos y materiales eficientemente, así como de manejar información biomédica en programas de prevención y tratamiento de la isoinmunización entre mujeres $\mathrm{Rh}$ negativo, ya sea en el ámbito hospitalario, en el estatal o en el nacional, o bien, en registros especiales o clínicas para mujeres Rh negativo. ${ }^{24}$

Como resultado de experiencias previas en México, ${ }^{25}$ en 1982 y a iniciativa del doctor Luis Sentíes Gutiérrez, se estableció formalmente, en el Instituto Nacional de Perinatología, la Clínica de Isoinmunización. Se constituyó con un grupo multidisciplinario, encargado de normar las acciones preventivas y terapéuticas para la atención de las mujeres Rh negativo, el feto y el recién nacido.

El objetivo de este trabajo es presentar los resultados de las actividades de prevención que se llevaron a cabo aplicando la $\gamma$-globulina anti-D en mujeres $\mathrm{Rh}$ negativo durante 1982-1995.

\section{Material y métodos}

En 1982, como parte del programa de isoinmunización materno-fetal que se manejaba en la Clínica, se inició el registro de las mujeres $\mathrm{Rh}$ negativo que acudieron para su atención médica al Instituto Nacional de Perinatología. Se elaboró un formato para el registro cronológico, donde se consignaron los datos demográficos y los antecedentes ginecobstétricos, inmunohematológicos y transfusionales de todas ellas. Se incluyeron los resultados de los estudios relacionados con los fenotipos del sistema Rh de la paciente y, en su caso, del cónyuge ${ }^{26}$ así como con las aplicaciones de la $\gamma$-globulina anti-D.

El programa contempla los criterios para la aplicación preventiva de $150 \mu \mathrm{g}$ de $\gamma$-globulina anti-D, por vía intramuscular, cuando se cubren los siguientes requisitos:

1. La madre debe ser Rh negativo.

2. No debe presentar evidencias de isoinmunización al $\mathrm{RhD}$, es decir, debe mostrar resultados 
negativos para el rastreo sérico de anticuerpos anti-D (aunque pudiera presentar algún otro anticuerpo antieritrocitario), en estudios realizados al menos 15 días antes del nacimiento del bebé.

3. El recién nacido debe ser Rh positivo y mostrar resultados negativos para anti-D, en la prueba directa de la antiglobulina humana (Coombs).

4. En caso de no poder identificar el grupo sanguíneo y el Rh fetal o neonatal, al recién nacido se debe considerar como $\mathrm{RhD}$ positivo.

El producto biológico se aplicó en las primeras 72 horas posteriores a la terminación del evento obstétrico, luego de haber documentado el cumplimiento de los criterios señalados anteriormente. La dosis se aumentaba a $300 \mu \mathrm{g}$ o más cuando había indicación médica precisa que orientara hacia hemorragia fetomaterna mayor o gestación múltiple.

Una vez aplicada la $\gamma$-globulina anti-D, la paciente acudía a hacerse una nueva valoración al menos doce semanas después de la aplicación de la dosis inicial, para repetir el rastreo de anticuerpos irregulares antieritrocitarios séricos. Si el resultado era negativo para la identificación de anti-D sérico materno, la acción preventiva se calificaba como exitosa. Si el resultado era positivo en la identificación de anticuerpos anti-D, el tratamiento se volvía a repetir cuando menos un mes después, ${ }^{27}$ si la detección inmunohematológica del anti-D sérico persistía, se consideraba que las acciones de prevención en ese caso habían fracasado, y si el resultado era negativo se les definía como exitosas.

El rastreo de anticuerpos irregulares se realizó mediante la técnica de hemaglutinación en tubo -que es ampliamente validada-, $, 18,19,22$ empleando la prueba de la antiglobulina humana y utilizando glóbulos rojos de fenotipo conocido, pertenecientes a sujetos de origen mestizo-mexicano; los glóbulos fueron proporcionados por el doctor Héctor Rodríguez Moyado y la Q.F.B. Elisa Quintanar García, del Banco Central de Sangre, del Centro Médico Nacional, centro hospitalario adscrito al Instituto Mexicano del Seguro Social. Se emplearon técnicas en las fases de salina rápida, salina a $22^{\circ} \mathrm{C}$, salina a $37^{\circ} \mathrm{C}$ y salina Coombs, incluyendo el control de la antiglobulina. ${ }^{19} \mathrm{La}$ utilización de los eritrocitos conocidos identifica la especificidad del anticuerpo con probabilidad menor a 0.05 .

En este artículo se define como mujeres Rh positivo (RhD) a quienes expresan el antígeno $\mathrm{D}$ perteneciente al sistema eritrocitario del $\mathrm{Rh}$, al provocar aglutinación eritrocitaria en presencia de antisuero antihumano, anti-D poliespecífico o con dos sueros anti-D de origen monoclonal. La ausencia en la reac- tividad con el suero anti-D identifica a las mujeres como Rh negativo. ${ }^{18,19,28}$

Con el fin de establecer la prevalencia de las pacientes $\mathrm{Rh}$ negativo en la población institucional, se obtuvieron los registros de todas las mujeres que ingresaron por primera vez al Instituto en el periodo de estudio. Se reportan las medidas de tendencia central de la población de estudio de acuerdo con el antecedente de sensibilización, acción preventiva mediante el biológico. Se registró la información sobre los grupos del sistema $A B O$, para establecer la supuesta protección de la incompatibilidad $\mathrm{ABO}$ de la isoinmunización; se utilizó la prueba de ji cuadrada, para establecer la diferencia entre la frecuencia de los grupos sanguíneos entre mujeres sensibilizadas y no sensibilizadas. Los resultados que se presentan incluyen a todas las pacientes independientemente del número de gestaciones, incompatibilidad $\mathrm{ABO}$ o antecedentes transfusionales.

\section{Resultados}

Durante el periodo que va de 1982 a 1995, ingresaron por primera vez al Instituto 100049 mujeres, de las cuales 4857 fueron identificadas como $\mathrm{Rh}$ negativo. En los datos agrupados por año, no se observó variación significativa; de este modo, se documentó una prevalencia institucional de pacientes $\mathrm{Rh}$ negativo de $4.85 \%$ (cuadro I). De las 4857 mujeres Rh negativo, $4228(87.1 \%)$ no presentaron isoinmunización al antígeno $\mathrm{D}$, es decir que en 629 casos (13.0\%) se registró la presencia de anticuerpos séricos de la especificidad anti-D, de tal forma que se documentó la isoinmunización materna al Rh.

\section{Cuadro I \\ Población Rh negativo y su condición de isoinmunización. Instituto Nacional de Perinatología, México, 1982-1995}

Condición de isoinmunización

No. (\%)

Total de pacientes de primera vez $(n) \quad 100049$

\begin{tabular}{lc}
\hline Pacientes Rh negativo & 4857 (4.85) \\
\hline Pacientes Rh negativo no isoinmunizadas & 4228 (87.0) \\
\hline Pacientes Rh negativo isoinmunizadas & $629(13.0)$
\end{tabular}

Momento en que se documentó la isoinmunización:

\begin{tabular}{lrl} 
A su ingreso & $542(86.2)$ \\
\hline Posterior a la $\gamma$-globulina & 22 & $(3.5)$ \\
\hline Posterior a su ingreso & (65) & 10.3
\end{tabular}

salud pública de méxico / vol.43, no.1, enero-febrero de 2001 
De las 629 pacientes Rh negativo isoinmunizadas, $542(86.2 \%)$ ya se encontraban isoinmunizadas desde antes de su ingreso al Instituto. En 22 casos (3.5\%), se pudo demostrar que la isoinmunización ocurrió a pesar de que estas mujeres fueron incluidas en el programa de prevención, al recibir la dosis adecuada y oportuna de la $\gamma$-globulina anti-D. Por último, aunque en 65 casos $(10.3 \%)$, los estudios iniciales en el rastreo de anticuerpos antieritrocitarios resultaron negativos, durante el curso de su seguimiento institucional, se reconoció la presencia de los anticuerpos antieritrocitarios anti-D, motivo por el cual ya no fueron candidatas a la aplicación de la g-globulina anti-D (cuadro I).

De las 4857 mujeres Rh negativo, 2605 (53.6\%) fueron incluidas en el programa de prevención de la isoinmunización, mediante la aplicación de la $\gamma$-globulina anti-D. En 2039 pacientes se aplicó una sola dosis y 475 casos recibieron hasta dos dosis en embarazos consecutivos. En las restantes 113 mujeres se administraron de 3 a 6 dosis (cuadro II).

En 22 pacientes se documentó la falla del programa de prevención con la $\gamma$-globulina anti-D, pues resultaron isoinmunizadas; 18 de ellas recibieron una sola dosis, y en cuatro casos, la isoinmunización se identificó después de la aplicación de dos dosis del biológico en eventos obstétricos diferentes. En estas pacientes se revisó la información disponible para establecer la causa probable del fracaso de la prevención. En cuatro casos se documentó la presencia de gestaciones múltiples, y se trató de mujeres que recibieron una sola dosis habitual del biológico. Los 18 casos restantes, presentaron patología obstétrica asociada, como enfermedad hipertensiva aguda asociada a la gestación, inserción baja de placenta y desprendimiento prematuro de placenta. Se intentó establecer la temporalidad entre la aplicación del biológico y la

Cuadro II

Número de dosis aplicadAs de $\gamma$-GLOBULINA ANTI-D. Pacientes del Instituto Nacional de Perinatología, México, 1982-1995

\begin{tabular}{lccrc} 
& \multicolumn{3}{c}{ Isoinmunización } & \multicolumn{2}{c}{ Número de dosis } \\
\cline { 3 - 5 } Total & $N b$ & $S i$ & No. & $\%$ \\
& & & 2039 & 77.6 \\
\hline 2 & 2021 & 18 & 475 & 18.1 \\
\hline 3 & 471 & 4 & 89 & 3.4 \\
\hline 4 & 89 & 0 & 20 & 0.2 \\
\hline $5-6$ & 20 & 0 & 4 & 0.2 \\
\hline Total & $2605(98.9 \%)$ & $22(0.8 \%)$ & 2627 &
\end{tabular}

identificación de la isoinmunización; en cuatro casos, al parecer la sensibilización ocurrió poco antes de la finalización del embarazo; en otros cinco casos se identificó la presencia del anticuerpo anti-D sérico, que persistió durante 2-5 meses, después de la aplicación del biológico, y en los 12 casos restantes se identificó la conversión serológica entre 1-7 meses después del embarazo anterior.

Las 1607 mujeres restantes no recibieron el biológico, lo que se debió a diferentes razones: se trataba de mujeres que tuvieron hijos Rh negativo; otras resolvieron su evento obstétrico fuera del Instituto, y también hubo pacientes que acudieron al Instituto por motivos ginecológicos.

Se compararon las determinaciones de los grupos sanguíneos y el sistema $\mathrm{ABO}$. El grupo con mayor prevalencia fue el $\mathrm{O}$, con $58.1 \%$, seguido del grupo A, con $30.4 \%$; le siguió el grupo B con $9.3 \%$, y por último, el grupo $\mathrm{AB}$, con $2.2 \%$. En el análisis de frecuencias, no se documentó diferencia estadísticamente significativa entre la frecuencia esperada de $\mathrm{ABO}$ en las mujeres no isoinmunizadas respecto de la frecuencia observada de $\mathrm{ABO}$ en las mujeres isoinmunizadas (datos no presentados).

\section{Discusión}

A principios de la década pasada, cuando ocurrió la transición para la aplicación prenatal de la $\gamma$-globulina anti-D, la incidencia de isoinmunización disminuyó de 9.1 a 2.2 por 1000 nacimientos. ${ }^{11,14}$ Con la aplicación prenatal, se redujo la sensibilización un 0.1 por 1000 nacimientos, mientras que la administración combinada, prenatal y posnatal, redujo la sensibilización a 0.03-0.18 por 1000 primigestas en riesgo. ${ }^{14}$

Llevar la isoinmunización a tasa cero es imposible, pues la literatura señala que los fracasos en la prevención de este padecimiento se deben, en una buena parte de los casos (84\%), al incumplimien de las guías o lineamientos del programa preventivo, ${ }^{7}$ y en menor frecuencia, a la ausencia de dicho programa. ${ }^{18,29}$ Por ejemplo, $62 \%$ de las mujeres con Rh negativo, cuyos hijos fallecieron por enfermedad hemolítica del recién nacido (EHRN), no habían recibido $\gamma$-globulina anti-D en embarazos previos de hijos Rh positivos. ${ }^{29}$

En el presente estudio, no todas las embarazadas $\mathrm{Rh}$ negativo fueron incluidas en el programa de prevención con el biológico pues, al igual que lo notificado en la literatura, sólo entre $60 \%$ y $89 \%$ de los casos resultaron elegibles. ${ }^{7}$ Se incluyó a mujeres sin considerar diferencias en la paridad o compatibilidad $\mathrm{ABO}$, aunque los programas de prevención con la $\gamma$-globulina anti-D tienen mayor costo-efectividad cuando se 
les aplica en mujeres Rh negativo primigrávidas, que cuando se emplea la misma dosis pero en todas las mujeres Rh negativo, independientemente del número de gestaciones. ${ }^{30}$

La dosis preventiva que se empleó fue de $150 \mu \mathrm{g}$, con la que se registró una sensibilización de $0.9 \%$ en las mujeres evaluadas. El empleo de esta dosis se determinó de acuerdo con el doctor Sentíes Gutiérrez, quien basó su criterio en la baja disponibilidad que existe del biológico en nuestro medio, ${ }^{23}$ lo que al fraccionar la dosis se protegería a un segmento más amplio de la población, y en que ante la presencia de hemorragia fetomaterna clínicamente importante, que ocurre en menos de $1 \%$ de todos los embarazos, ni la dosis propuesta de $150 \mu \mathrm{g}$, ni la dosis estándar de $300 \mu \mathrm{g}$, lograrían una prevención efectiva. ${ }^{22}$

Con las dosis de $100 \mu \mathrm{g}$ o $200 \mu \mathrm{g}$ de $\gamma$-globulina anti-D, administradas a primigrávidas en riesgo, el desarrollo de anti-D se notifica en el $0.10 \%$ de las mujeres tratadas. ${ }^{31}$ Sin embargo, la evidencia señala que las dosis con mayor costo-efectividad varía entre $200 \mu$, y $300 \mu g^{20,32}$ pues propicia una reducción significativa en la incidencia de la isoinmunización a los seis meses posparto (RR 0.04, IC95\% 0.02-0.06) y en el siguiente embarazo (RR 0.12, IC95\% 0.07-0.23).

Es una práctica difundida el empleo prenatal de una dosis estándar de $\gamma$-globulina anti-D $(300 \mu \mathrm{g})$ en la semana $28,{ }^{11}$ o bien, el uso de dosis más bajas $(100 \mu \mathrm{g} o$ $250 \mu \mathrm{g}$ la semana 28 y en la 34 de embarazo, para reducir la tasa de isoinmunización a menos de la unidad porcentual. ${ }^{30,33,34} \mathrm{Sin}$ embargo, la evidencia no es concluyente debido a los errores metodológicos en el diseño de los estudios publicados, ${ }^{21,22}$ incluidas variables como el grupo de gestantes que debe ser el blanco primario (las primigrávidas) ${ }^{27} \mathrm{o}$ la dosis empleada. $^{33}$

A pesar de las dificultades económicas que existen en los países subdesarrollados, los programas preventivos de la isoinmunización al $\mathrm{Rh}$ representan un menor costo que las acciones terapéuticas ante la isoinmunización y la EHRN resultante, aunque el elevado costo del biológico y su baja disponibilidad son grandes limitantes. ${ }^{18,35}$

Con la intención de establecer las prioridades en la prevención de la isoinmunización al Rh, se analizó la mejor evidencia disponible en la literatura y se evaluó el nivel de efectividad ${ }^{20,21}$ respecto a las intervenciones médicas fundamentales, ${ }^{18,22}$ así, se obtuvieron las siguientes recomendaciones (cuadro III):

- Hay buena evidencia sobre la práctica de identificación del grupo sanguíneo y el rastreo de anticuerpos irregulares en la consulta prenatal o en el posparto inmediato, si se tiene un neonato $\mathrm{RhD}$ positivo, anteriormente llamado $\mathrm{RhD}$ o variante $\mathrm{Du}$ débil (nivel I, recomendación A). En este renglón se incluye a las mujeres en situación de aborto. ${ }^{36,37}$

- Es aceptable la evidencia (nivel II-2, recomendación B), que apoya el rastreo de anticuerpos (Coombs indirecto) en las semanas 24 a 28 de gestación. ${ }^{19,28}$

- Aunque hay ventaja inicial (recomendación B), no son concluyentes los estudios sobre la práctica uniforme en el empleo prenatal de la $\gamma$-globulina anti-D. ${ }^{12,14,18,31,33}$

- Es aceptable la evidencia que recomienda el rastreo luego del aborto inducido (nivel III, recomendación B) y de la amniocentesis (nivel II-2, recomendación B), ante la probabilidad de hemorragia fetomaterna. ${ }^{5,38,39}$

- Es pobre la evidencia que recomienda la inclusión o exclusión del rastreo de anticuerpos, ante la aspiración de vellosidades coriales. ${ }^{5,22}$

- Es pobre la evidencia (nivel III, recomendación C) que recomienda la inclusión o la exclusión del rastreo de anticuerpos para otras complicaciones obstétricas o procedimientos invasores. ${ }^{18,40}$

- Aunque el beneficio económico, en términos de administración del financiamiento, es evidente (nivel III, recomendación B), los datos observacionales apuntan a recomendar que las pacientes $\mathrm{Rh}$ negativo se atiendan en centros especializados de acuerdo con programas específicos. ${ }^{24}$

Los programas de prevención de la isoinmunización materna al $\mathrm{RhD}$, que incluyen el empleo de la $\gamma$ globulina anti-D, forman parte de las actividades preventivas más exitosas en el ámbito mundial, con un alto impacto en el financiamiento para la salud. ${ }^{30}$ Sin embargo, en nuestro país deben enfrentar graves problemas de disponibilidad del biológico, pues apenas $60-80 \%$ de la población podría recibir esta protección. ${ }^{23}$ Tal situación constituye un motivo para considerar como opción la reducción de la dosis de $\gamma$-globulina anti-D, de $300 \mu \mathrm{g}$ a $150 \mu \mathrm{g}$, con resultados favorables. Además, es necesario insistir en la promoción y difusión de los programas preventivos de la isoinmunización.

\section{Referencias}

1. Grunbaum BW, Selvin S, Pace N, Black DM. Frequency distribution and discrimination probability of twelve protein genetic variants in human blood as functions of race, sex, and gene. J Forensic Sci 1978;23:577-578. 


\section{Cuadro III}

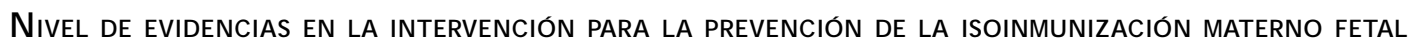

Maniobra

- Identificación prenatal del grupo sanguíneo $A B O$ y RhD, y rastreo de anticuerpos irregulares en la primera visita prenatal

- Repetir rastreo de anticuerpos dentro de las 72 horas siguientes al parto de un neonato RhD positivo

- Repetir el rastreo de anticuerpos entre la semana 24-28 de la gestación, en la madre no isoinmunizada

- Prevención de la isoinmunizac posparto mediante la $\gamma$-globulina anti-D

Efectividad

Nivel de evidencia (referencia)

Recomendación

- Inter vención. Administración de $300 \mu \mathrm{g}$ de $\gamma$-globulina anti-D vía IM a mujeres no sensibilizadas dentro de las primeras 72 horas luego de haber tenido un hijo RhD (incluidos los casos con muerte intrauterina)
- Estudios clínicos aleatorios [I] $]^{36,37}$ Hay evidencia sustancial que apoya tal recomendación para el rastreo de anticuerpos en la visita antenatal y en el posparto [A]
- Profilaxis prenatal con $\gamma$-globulin anti-D

\section{- Práctica útil para seleccionar ade- · Estudios observacionales y opinión • Acción aparentemente benéfica, cuadamente a las candidatas a pro- filaxis con $\gamma$-globulina anti-D pero sin evidencia sustancial [C] \\ - Se tiene evaluada la dosis estándar Revisión sistemática [I] $]^{20}$ de $300 \mu \mathrm{g}$ en el puerperio inme- diato \\ - Existe evidencia sustancial que apoya tal recomendación [A] \\ - Estudios clínicos iniciales, favora- bles, aunque en la revisión sistemáti- ca no muestra diferencia [C] $\gamma$-globulina anti-D, en diferentes dosis, en las semanas 28 y 34 , com- paradas con el empleo posparto \\ - Estudios controlados no aleatorios [II-1] 12,14,33 \\ - Estudios clínicos no aleatorios [II- $2]^{31}$ \\ - Estudios clínicos controlados [II- 3] $]^{18,31,33}$ \\ - Revisión sistemática [I] ${ }^{21}$}

- Repetir el rastreo de anticuerpos antes del aborto inducido, amniocentesis u otra complicación obstétrica 0 procedimiento en donde haya posibilidad de hemorragia feto-materna
- La administración profiláctica de - Aborto inducido: serie de casos $300 \mu \mathrm{g}$ de lg anti-D luego del abor- $\quad[111]^{38,39}$

to 0 amniocentesis $(50 \mu \mathrm{g}$ si ocu- - Amniocentesis, pruebas clínicas conrre antes de la semana 13), reduce troladas no aleatorias [II-2 $]^{5,6}$ la incidencia de isoinmunización

- AlII] $]^{38,39}$
troladas no aleatorias [II-2 $]^{5,6}$

- Hay razonable evidencia que apoya la recomendación de que el rastreo de anticuerpos se efectúe con el aborto inducido 0 amniocentesis [B]

- Tasas similares de sensibilización fueron encontradas tanto en el grupo de estudio como en el control, luego de la biopsia de vellosidades coriales

- Estudios clínicos aleatorios [I] $]^{5,22}$

- Hay insuficiente evidencia que documente la inclusión o exclusión del rastreo de anticuerpos irregulares en los casos sometidos a biopsia de vellosidades coriales [C]

- Se debe efectuar la prevención en otros procedimientos o complicaciones obstétricas, en donde se pueda sospechar la probabilidad de hemorragia feto-materna

- Estudios clínicos no controlados, opinión de expertos [IIII] $]^{18,40}$

- En amenaza de aborto se debe de efectuar prevención [IIII] ${ }^{18}$

- Hay insuficiente evidencia acerca de rastreo de anticuerpos irregulares en otras complicaciones obstétricas, procedimientos o pronósticos [C]

\footnotetext{
- Se debe efectuar la prevención en aquellas pacientes en riesgo que muestran paridad satisfecha y desean algún método de esterilización - Se debe mantener la protección • 0 pinión de expertos [II-3, III]

- Hay pobre evidencia que demuessobre el futuro transfusional de la mujer, empleando el biológico tre una ventaja 0 desventaja de la aplicación de la $\gamma$-globulina en estas condiciones [C]

- Manejo de las mujeres Rh negativo sensibilizadas en centros especiales de atención

- Se deben concentrar recursos y • Estudios observacionales [IIII $]^{24}$

- Acción probablemente benéfica, experiencias en centros especializados pero que refleja una ganancia coherente en términos de atención de las pacientes y de financiamiento para la salud $[B]$
}

Estas recomendaciones se basaron en las guías establecidas por U. S. Preventive Services Task Force, ${ }^{22}$ investigando MED LIN E de 1989 a enero del 2000 , con el motor de búsqueda de PubMed con las palabras clave: Rh-Hr, isoinmunización, anti-D, amniocentesis, transfusión intrauterina, erythroblastosis fetal, enfermedad hemolítica. Las recomendaciones se estratificaron como: A: evidencia que apoya la recomendación para ser específicamente considerada. B: razonable evidencia que apoya la recomendación para ser específicamente considerada. C: pobre evidencia para documentar la inclusión o exclusión de la condición, pero la recomendación puede ser hecha en otros ámbitos. D: razonable evidencia que apoya que la recomendación pueda ser excluida de tal consideración, y E: buena evidencia que apoya que la recomendación puede ser excluida de tal consideración. Para la calidad de la evidencia se emplearon cinco niveles. I: evidencia de al menos inapropiado estudio clínico controlado aleatorio. II-1: evidencia de un estudio clínico controlado bien diseñado, no aleatorio, preferiblemente de más de un grupo de investigación. II-2: evidencia de al menos un estudio clínico bien diseñado, cohorte 0 casos-control, preferiblemente de más de un grupo de investigación. II-3: evidencia de comparación en tiempo o lugar, con o sin intervención. Dramáticos resultados en estudios no controlados también pueden ser considerados. III: 0 pinión de autoridades, basada en experiencias clínicas 0 estudios descriptivos, 0 bien, comité de expertos 
2. Lisker R. Estructura de la población genética en México. México, DF.: Salvat, 1982.

3. Huchcroft S, G unto P, Bowen T. Compliance with postpartum Rh isoimmunization prophylaxis in Alberta. Can Med Assoc J 1985;133:871-875.

4. Von Stein GA, Munsick S, Stiver K, Ryder K. Fetomaternal hemorrhage in threatened abortion. 0 bstet Gynecol 1992;79:383-386.

5. Smidt-Jenson S, Philip J. Comparison of transabdominal and transcervical CVS and amniocentesis: sampling success and risk. Prenat Diagn 1991;11:529-537.

6. D affos F, C apella-Pavlovsky M, Forestier F. Fetal blood sampling during pregnancy with use of a needle guided by ultrasound: A study of 606 consecutive cases. Am J 0 bstet Gynecol 1985;153:655-660.

7. Howard HL, Martlew VJ, MCFadyen IR, Clarke CA. Preventing Rhesus D haemolytic disease of the newborn by giving anti-D immunoglobulin: Are the guidelines being adequately followed? Br J 0 bstet G ynaecol 1997;105: 37-41.

8. Beaulieu MD. Screening for D (Rh) sensitization in pregnancy. En: Prenatal and perinatal preventive care. 0 ttawa, 1980:116-124.

9. Baptista GHA, Rosenfeld MF, Pérez PJ, Q uintanar GE. Anticuerpos irregulares antieritrocitarios fuera del sistema $A B O$ en el periodo perinatal. Bol Med Hosp Infant Mex 1991;48:814-820.

10. Urbiniak SJ. $R h(D)$ haemolytic disease of the newborn: The changing scene. B M J 1985;291:4-6.

11. Bowman JM. Controversies in Rh profilaxis. W ho needs Rh immunoglobulin and when should it be given? Am J 0 bstet Gynecol 1985; 151: 289-294

12. MCSweeney E, Kirkham J, Vinall P, Flanagan P. An audit of anti-D sensitisation in Yorkshire. Br J O bstet Gynaecol 1998;105:1091-1094.

13. W ithfield CR, Raafat A, U rbaniak SJ. Underreporting of mortality from RhD haemolytic disease in Scotland and its implications: Retrospective review. BMJ 1997;315:1504-1505.

14. Bowman JM, Pollock JM. Rh immunization in Manitoba: Progress in prevention and management. Can Med Assoc J 1983;129:4 343-345.

15. Bowman JM, Chown $B$, Lewis M, Pollock J. Rh isoimmunization, Manitoba, 1963-75. Can Med Assoc | 1977;116:282-284.

16. Centers for D isease Control. Rh Hemolytic disease - C onnecticut, United States, 1970-1979. MMW R Morb Mortal W kly Rep 1981;30:13-15.

17. Baskett TF, Parsons ML, Peddle LJ. The experience and effectiveness of the N ova Scotia Rh program, 1964-84. Can Med Assoc J 1986;134: $1259-1261$.

18. ACO G practice bulletin. Prevention of Rh D alloimmunization. $\mathrm{N}$ umber 4, May 1999 (replaces educational bulletin N umber 147, 0 ctober 1990). Clinical management guidelines for obstetrician-gynecologists. American College of $O$ bstetrics and Gynecology. Int I Gynaecol O bstet 1999;66: 63-70.

19. American Association of Blood Banks. Technical Manual. $12^{\text {th }}$ ed. Bethesda, Maryland: A merican Association Blood Banks, 1996.

20. Crowther $C$, Middleton P. Anti-D administration after childbirth for preventing Rhesus alloimmunization (Cochrane Review). 0 xford: U pdate Software, 2000.

21. Crowther C, Keirse MJNC. Anti-D administration in pregnancy for preventing Rhesus alloimmunization (Cochrane Review). Cochrane Library Issue 1. 0 xford: Update Software, 2000.
22. U.S. Preventive Services Task Force. G uide to clinical preventive services: An assessment of the effectiveness of 169 interventions. Baltimore (Md): W illiams \& W ilkins, 1989:221-224.

23. Zavala C, Salamanca F. Mothers at risk of alloimmunization to the Rh (D) antigen and availability of gamma-globulin at the Mexican Institute of Social Security. Arch Med Res 1996;27:373-376.

24. W hittle MJ. Antenatal serology testing. PACE review N 0 97/02. Londres: Royal College of O bstetricians and Gynecologist, 1997.

25. Cepeda AG, Sentíes L, Fiorelli FE, G arcete JA. Prevention of post-partum Rh-factor isoimmunization (experience of the Hospital 1st Gynecological-O bstetrical of the Mexican Institute of Social Security). Ginecol 0 bstet Mex 1975;37:83-91.

26. Baptista GHA, Rosenfeld MF. Fenotipo del sistema Rh-Hr de los cónyuges de pacientes Rh negativo. Perinatol Reprod Hum 1993;7:44-48.

27. W itter FR, Shirey RS, N icol SL, N ess PM. Postinjection kinetics of antepartum Rh immune globulin. Am J O bstet Gynecol 1990;163:784-786. 28. British Committee for Standars in Haematology. Guidelines for blood grouping and red cell antibody testing during pregnancy. Transf US Med 1996;6:71-74.

29. Hughes RG, Craig JI, Murphy W G, G reer IA. C auses and clinical consequences of Rhesus (D) haemolytic disease of the newborn: A study of a Scottish population, 1985-1990. Br J O bstet Gynaecol 1994;101:297-300. 30. Vick S, C airns J, U rbaniak S, W hitfield C, Raafat A. C ost-effectiveness of antenatal anti-D prophylaxis. Health Econ 1996;5:319-328.

31. Lee $D$, Rawlinson VI. Multicenter trial of antepartum low-dose anti-D immunoglobulin. Transfus Med 1995;5:15-19.

32. Moncharmont $P$, Juron D upraz F, Vignal M, Rigal D, Meyer F, D ebeaux $P$. Haemolytic disease of the newborn infant. Long term efficiency of the screening and the prevention of alloimmunization in the mother: Thirty years of experience. Arch Gynecol 0 bstet 1991;248:175-180.

33. Trolle B. Prenatal Rh-immune prophylaxis with 300 micrograms immune globulin anti-D in the $28^{\text {th }}$. week of pregnancy. Acta 0 bstet $G$ ynecol Scand 1989;68:45-47.

34. MacKenzie IZ, Bowell P, G regory H, Pratt G, Guest C, Entwistle CC. Routine antenatal Rhesus $D$ immunoglobulin prophylaxis: The results of a prospective 10 year study. Br J O bstet Gynaecol 1999;106:492-497.

35. Joseph KS. C ontrolling Rh haemolytic disease of the newborn in India. Br J O bstet Gynaecol 1991;98:369-377.

36. Chown B, Duff AM, James J, et al: Prevention of primary Rh immunization: First report of the Western Canadian Trial, 1966-1968. Can Med Assoc I 1969;100:1021-1024.

37. Pollack W, Gorman JG, Freda VJ. Results of clinical trials of RhoGAM in women. Transfusion 1968:8:151-153.

38. Simonovits I. Efficiency of anti-D IgG prevention after induced abortion. Vox Sang 1974;26:361-367.

39. Simonovits I, Timar I, Bajtai G: Rate of Rh immunization after induced abortion. Vox Sang 1980;38:161-164.

40. Lee D, Contreras M, Robson SC, Rodeck CH, W hittle MJ. Recommendations for the use of anti-D immunoglobulin for Rh prophylaxis. British Blood Transfusion Society and the Royal College of 0 bstetricians and Gynaecologists. Transfus Med 1999;9:93-97. 